\title{
Markov Chains and Optimality of the Hamiltonian Cycle
}

\author{
Nelly Litvak \\ Faculty of Electrical Engineering, Mathematics and Computer Science, Department of Applied Mathematics, \\ University of Twente, P.O.Box 513, 7500 AE, Enschede, The Netherlands \\ email: N.Litvak@math.utwente.nl http://wwwhome.math.utwente.nl/ litvakn/ \\ Vladimir Ejov \\ Centre of Industrial and Applied Mathematics, School of Mathematics and Statistics,University of South \\ Australia, Mawson Lakes, SA 5095, Australia \\ email: Vladimir.Ejov@unisa.edu.au \\ http://www . unisanet. unisa.edu.au/Staff/Homepage.asp?Name=Vladimir.Ejov
}

\begin{abstract}
We consider the Hamiltonian cycle problem (HCP) embedded in a controlled Markov decision process. In this setting, HCP reduces to an optimization problem on a set of Markov chains corresponding to a given graph. We prove that Hamiltonian cycles are minimizers for the trace of the fundamental matrix on a set of all stochastic transition matrices. In case of doubly stochastic matrices with symmetric linear perturbation, we show that Hamiltonian cycles minimize a diagonal element of a fundamental matrix for all admissible values of the perturbation parameter. In contrast to the previous work on this topic, our arguments are primarily based on probabilistic rather than algebraic methods.
\end{abstract}

Key words: Markov chains; Hamiltonian cycle; Fundamental matrix; Singular perturbation

MSC2000 Subject Classification: Primary: 60J10; Secondary: 05C45, 15A51

OR/MS subject classification: Primary: probability, Markov processes; Secondary: networks/graphs, theory; probability, renewal processes

1. Introduction. This paper continues the line of research introduced in Filar and Krass [15] where the Hamiltonian cycle problem (HCP) was embedded in a controlled Markov decision process. This approach was continued by Chen and Filar [10] and by Feinberg [13]. Further results were obtained by Filar and Liu [17], Andramonov et al. [2], Filar and Lasserre [16], Ejov et al. [11, 12], and Borkar et al. $[7,8]$ on solving the Hamiltonian cycle problem by translating it into an optimization problem on a set of Markov chains contained in a Markov decision process.

Consider a directed graph $\mathcal{G}$ with the node set $S$ and the arc set $\mathcal{A}$. A Markov decision process $\Gamma$ can be associated with the graph $\mathcal{G}$ as follows. The set of $n$ nodes in $\mathcal{G}$ represents the finite state space $\mathcal{S}=\{1,2, \ldots, n\}$ on a discrete time $(t=0,1,2, \ldots)$. Let $X_{t}$ be the state at time $t$. For each state $i$, the actions are simply arcs $(i, j)$ from the corresponding node. The set of possible actions in state $i$ is then $\mathcal{A}(i)=\{j \mid(i, j) \in \mathcal{A}\}$. Let $A_{t}$ be the action at time $t$. Further, assume that the transition probabilities are given by $p(j \mid i, a)=\mathbb{P}\left(X_{t+1}=j \mid X_{t}=i, A_{t}=a\right)=\delta_{j a}$ for all $i, j \in S, a \in \mathcal{A}(i)$, where $\delta_{j a}$ is a Kronecker delta. Now consider a class $\mathcal{F}$ of randomized stationary policies, and let $p_{i j}$ be the probability of choosing an action $j(\operatorname{arc}(i, j))$ if the current state is $i$. Of course, $\sum_{j=1}^{n} p_{i j}=1$ and if $(i, j) \notin \mathcal{A}$, then, by default, $p_{i j}=0$. Under the above assumptions, a policy $P=\left(p_{i j}\right)$ constitutes a transition matrix of the Markov chain $\left(X_{t}\right)$ on $\mathcal{G}$.

The idea of the approach pioneered in Filar and Krass [15] is to formulate an optimization problem on a class of transition matrices (policies) $P \in \mathcal{F}$, such that an objective function is minimized (or maximized) on matrices that induce a Hamiltonian cycle. Then HCP can be reduced to solving a corresponding optimization problem. Furthermore, there is a freedom of choosing the objective function and the transition probabilities $p(j \mid i, a), i, j \in S, a \in \mathcal{A}(i)$, so that the optimization problem could resolve easier, thus providing an efficient way for solving HCP.

The objective function commonly used in the above mentioned literature is the top diagonal element of a fundamental matrix defined as follows. Let $P$ be a transition matrix of a Markov chain $\left(X_{t}\right)$ on $\mathcal{S}$. An ergodic projection $\Pi$ is defined as the limit Cesaro-sum matrix

$$
\Pi=\lim _{T \rightarrow \infty} \frac{1}{T+1} \sum_{t=0}^{T} P^{t} .
$$

If $P$ induces only one ergodic class then $\Pi$ consists of identical rows $\pi=\left(\pi_{1}, \cdots, \pi_{n}\right)$, where $\pi$ is the 
unique stationary distribution of the Markov chain, satisfying

$$
\pi P=\pi, \quad \sum_{i=1}^{n} \pi_{i}=1
$$

The fundamental matrix $G=\left(G_{i j}\right)$ is given by

$$
G \stackrel{\text { def }}{=}(I-P+\Pi)^{-1}=\lim _{\beta \rightarrow 1^{-}} \sum_{t=0}^{\infty} \beta^{t}(P-\Pi)^{t} .
$$

It has been noted by Filar and Liu [17] that the top left hand corner element $G_{11}$ is minimized on Hamiltonian cycles over the class of deterministic policies singularly perturbed with asymmetric linear perturbation for sufficiently small values of the perturbation parameter (see Filar [14] for more detail). Ejov et al. [12] proved this result with precise bounds for the perturbation parameter and conjectured that within these boundaries, Hamiltonian cycles minimize $G_{11}$ over the entire class $\mathcal{F}$ of stochastic matrices.

An interesting subclass of $\mathcal{F}$ is a set $\mathcal{D} S$ of doubly stochastic matrices. A matrix $P$ is doubly stochastic if each row and each column of $P$ sums up to unity. In Borkar et al. [7] the minimization of $G_{11}$ was considered over the set $\mathcal{D} S$, and a probabilistic interpretation of $G_{11}$ was given. Further, it was shown in Borkar et al. [8] that for symmetric linear perturbation, minimizing $G_{11}$ over $\mathcal{D} S$ is equivalent to minimizing $\operatorname{Var}\left(\tau_{1}\right)$, where $\tau_{1}$ is the time between two successive visits to state 1 . Moreover, it was shown that for sufficiently small values of the perturbation parameter, the minimum is achieved on a matrix that induces a Hamiltonian cycle. However, no explicit bound for the perturbation parameter was given. In Section 5 we use a Renewal Theory argument to show that this result holds in fact for any admissible value of the perturbation parameter.

The Markov chain formulation of the HCP allows to naturally employ Probability Theory for tackling a classical problem from Discrete Mathematics. Consequently, probabilistic methods play a central role in the present paper. In this regard, we make a substantial step forward compared to the previous work where algebraic techniques were heavily used. We provide the analysis for the objective function $\operatorname{Tr}[G]$. Compared to $G_{11}$, the trace has the benefit of being invariant under permutations of the nodes of the graph. The connection between $G_{11}$ and the cycle length $\tau_{1}$ is well-known in literature (see Aldous and Fill [1], Borkar et al. [8], Hunter [19]). In Section 2 we elaborate on the relations between $\operatorname{Tr}[G]$, mixing times and variability of cycle lengths. Hunter [19] proved that the matrix inducing a Hamiltonian cycle minimizes $\operatorname{Tr}[G]$ over a set of irreducible transition matrices. We extend this result to the set of all stochastic matrices. In Section 3, we discuss the trade-off between minimizing the trace and the diagonal element of $G$. For doubly stochastic matrices, these two objective functions are equivalent, whereas for arbitrary stochastic matrices proving such equivalence remains an open problem.

Sections 4 and 5 are devoted to the matrices with symmetric linear perturbation. Such perturbation ensures irreducibility of the transition matrix and provides important advantages from computational point of view. Due to these features, matrices with linear perturbation play important role in applications, Google PageRank being perhaps a most remarkable example. Our results on perturbed transition matrices can be directly used for solving the optimization problem, which implies the solution of HCP. In particular, the extension of results from Borkar et al. [8] to any value of the perturbation parameter gives important advantages for developing fast numerical algorithms. Moreover, we believe that the employed probabilistic techniques constitute a plausible and promising tool for tackling optimization problems related to perturbed Markov chains.

2. Minimizing mixing times and variability. We start with some common definitions and notations. Let $P=\left(p_{i j}\right)$ be a transition matrix of a Markov chain $\left(X_{t}\right)$ with a finite state space $\mathcal{S}=\{1,2, \ldots, n\}$ on a discrete time $(t=0,1,2, \ldots)$. If the Markov chain has only one ergodic class, then denote by $\pi$ the invariant distribution.

Consider a deviation matrix $Z=\left(Z_{i j}\right)$, which is closely related to the fundamental matrix $G$ and is defined formally as follows:

$$
Z=[I-P+\Pi]^{-1}-\Pi=G-\Pi .
$$

In case when the matrix $P$ is aperiodic, $Z$ can be written as

$$
Z=\sum_{t=0}^{\infty}\left[P^{t}-\Pi\right] .
$$


An element $Z_{i j}$ here can be seen as an average difference in the number of visits to $j$ on $[0, \infty)$ between the chain started at $i$ and the chain started from the stationary distribution. If $P$ is periodic then the above equation does not make sense since $P^{t}$ does not converge to a limit as $t \rightarrow \infty$ and thus the above sum does not exist. To deal with periodicity, we define the matrix $Z$ via Cezaro limits:

$$
Z=\lim _{T \rightarrow \infty} \frac{1}{T+1} \sum_{t_{0}=0}^{T} \sum_{t=0}^{t_{0}}\left[P^{t}-\Pi\right]
$$

The matrix $Z$ in (4) is well defined and is equivalent to (2) for any finite state discrete-time Markov chain.

Denote by $\tau_{i}$ the time interval between two successive visits to state $i \in \mathcal{S}$. The cycle length $\tau_{i}$ is well defined for any recurrent state $i \in \mathcal{S}$. Further, the hitting times are introduced as $T_{i}=\min \left\{t \geq 0: X_{t}=\right.$ $i\}, i \in \mathcal{S}$. In words, $T_{i}$ is the epoch when the Markov chain $\left(X_{t}\right)$ reached $i$ for the first time. If state $i$ can not be reached then $T_{i}$ equals $+\infty$. Throughout the paper, we will use a lower index to indicate the initial distribution, e.g.,

$$
\mathbb{P}_{i}\left[T_{j}=k\right]=\mathbb{P}\left[T_{j}=k \mid X_{0}=i\right], \quad \mathbb{E}_{i}\left[T_{j}\right]=\mathbb{E}\left[T_{j} \mid X_{0}=i\right], \quad \mathbb{E}_{\pi}\left[T_{j}\right]=\sum_{i=1}^{n} \pi_{i} \mathbb{E}_{i}\left[T_{j}\right]
$$

In this section we will highlight the connections between the deviation matrix $Z$, variability of $\tau_{i}$ 's, hitting times, and the Hamiltonian cycle problem.

2.1 Irreducible chains. In the irreducible case, there is a close relation between the diagonal elements of the matrix $Z$ and hitting times $T_{i}, i \in \mathcal{S}$. Lemma 11 in Chapter 2 of Aldous and Fill [1] states that

$$
\pi_{i} \mathbb{E}_{\pi}\left(T_{i}\right)=Z_{i i}
$$

Combining it with formula (21) in the same chapter, we obtain

$$
\mathbb{E}_{i}\left[\tau_{i}^{2}\right]=\frac{2 \mathbb{E}_{\pi}\left[T_{i}\right]}{\pi_{i}}+\frac{1}{\pi_{i}}=\frac{2 Z_{i i}}{\pi_{i}^{2}}+\frac{1}{\pi_{i}}
$$

Let $c_{i}^{2}=\mathbb{E}\left[\tau_{i}^{2}\right] /\left(\mathbb{E}\left[\tau_{i}\right]\right)^{2}$ be the coefficient of variation of $\tau_{i}$. Since $\mathbb{E}\left[\tau_{i}\right]=1 / \pi_{i}$, it follows from (6) that

$$
c_{i}^{2}=\pi_{i}^{2} \mathbb{E}_{i}\left[\tau_{i}^{2}\right]=2 Z_{i i}+\pi_{i}, \quad i=1, \ldots, n .
$$

Summing over $i$, we get

$$
\sum_{i} c_{i}^{2}=2 \sum_{i} Z_{i i}+1
$$

and thus, minimizing any of the two sums above is equivalent. This gives us an alternative probabilistic proof of the next proposition, which has been recently obtained in Hunter [19] by linear algebraic methods.

Proposition 2.1 On a set of irreducible transition matrices, the objective function $\operatorname{Tr}[Z]=\sum_{i} Z_{i i}$ achieves its minimal value $(n-1) / 2$, or, equivalently, $\sum_{i} c_{i}^{2}$ achieves its minimal value $n$ at matrix $P$ if and only if $P$ induces a Hamiltonian cycle.

Proof. If $P$ is a Hamiltonian matrix, then for any $i \in \mathcal{S}$, we have $\tau_{i}=n=$ const implying that $c_{i}^{2}$ takes its minimal possible value 1 . Thus, the Hamiltonian cycle provides $\sum_{i} c_{i}^{2}=n$ which is the minimal possible value of this sum. Moreover, no other irreducible matrix has such property. Indeed, in any irreducible non-Hamiltonian Markov chain, there exists a state $j$ such that $\tau_{j}$ is not deterministic, implying $c_{j}^{2}>1$, and, consequently, $\sum_{i} c_{i}^{2}>n$.

The fact that $Z_{i i}$ is related to $c_{i}^{2}$ can be also deduced from the Renewal Theory. Let Markov chain start at state $i \in \mathcal{S}$ and consider a renewal process where renewal instants are successive visits to $i$. The interval between two renewals is distributed as $\tau_{i}$. Define $N_{i}(t)$ as the number of renewals on the interval $(0, t]$. Let $\gamma_{i}(t)$ be the excess time, i.e. the time interval between $t=0,1, \ldots$ and the nearest visit to $i$ after time $t$. Then we can use the following well-known formula which is a direct consequence of the Wald's equation (see e.g. Ross [22], p. 106):

$$
\mathbb{E}\left[\tau_{i}\right]\left(\mathbb{E}_{i}\left[N_{i}(t)\right]+1\right)=t+\mathbb{E}\left[\gamma_{i}(t)\right], \quad t \geq 0 .
$$


Hence, in the aperiodic case, for any $i \in \mathcal{S}$, we derive

$$
\begin{aligned}
& Z_{i i}:=\sum_{t=0}^{\infty}\left[\left(P^{t}\right)_{i i}-\pi_{i}\right] \\
& =\lim _{t \rightarrow \infty}\left(\mathbb{E}_{i}\left[N_{i}(t)\right]+1-(t+1) \pi_{i}\right) \\
& =\pi_{i} \lim _{t \rightarrow \infty} \mathbb{E}\left[\gamma_{i}(t)\right]-\pi_{i} \quad \text { from (8) and } \mathbb{E}\left[\tau_{i}\right]=1 / \pi_{i} \\
& =\pi_{i}\left(\frac{\mathbb{E}\left[\tau_{i}^{2}\right]}{2 \mathbb{E}\left[\tau_{i}\right]}+\frac{1}{2}\right)-\pi_{i} \quad \text { using } \lim _{t \rightarrow \infty} \mathbb{E}\left[\gamma_{i}(t)\right] \text { in discrete time } \\
& =\frac{1}{2}\left(c_{i}^{2}-\pi_{i}\right)
\end{aligned}
$$

which is the same as (7). If $i$ is a periodic state, we obtain the same result using Cezaro limits as in (4).

Let us now consider a hitting time $T_{\pi}$ of a state randomly sampled from a stationary distribution. The expectation of $T_{\pi}$ represents one of the simplest versions of 'time to mixing', that is, the time needed to reach stationarity. We refer to Lovász and Winkler [21] and Chapter 4 of Aldous and Fill [1] for a detailed treatment of various mixing time concepts. The relation between $\operatorname{Tr}[Z]$ and the mixing time $T_{\pi}$ is given by the Random Target Lemma (see Aldous and Fill [1], Chapter 2, Corollary 13) as follows:

$$
\mathbb{E}_{i}\left[T_{\pi}\right]=\sum_{j} \pi_{j} \mathbb{E}_{i}\left[T_{j}\right]=\operatorname{Tr}[Z], \quad \text { for any } i=1, \ldots, n \text {. }
$$

The striking fact that $\mathbb{E}_{i}\left[T_{\pi}\right]$ does not depend on $i$ is well-known in literature. Implications of this result have been studied in detail by Hunter [19]. We would like to emphasize that Proposition 2.1 and the Random Target Lemma reveal the connection between mixing times and variability of cycles in Markov chains. Such connection has been also observed in Chapter 2 of Aldous and Fill [1].

Coming back to the Hamiltonian cycle problem, we can now use Proposition 2.1 to state that a transition matrix inducing a Hamiltonian cycle minimizes $\operatorname{Tr}[Z]$ on a set of irreducible Markov chains that correspond to a given graph. However, the irreducibility assumption is not plausible because it can not be written as a set of simple constraints on transition probabilities. Thus, it is relatively difficult to incorporate irreducibility into an algorithm solving an optimization problem. In the next section we resolve this issue by generalizing Proposition 2.1 to Markov chains with arbitrary structure.

2.2 Chains with arbitrary structure. First, assume that $P$ is such that there is a class of transient states $\mathcal{C}_{0}$ and a class of ergodic states $\mathcal{C}_{1}$. In this case, the matrix $Z$ is defined by (4) but for $i \in \mathcal{C}_{0}$, the quantity $c_{i}^{2}$ is not well defined, and equations (5) and (7) do not hold. However, the next proposition shows that $\operatorname{Tr}[Z]$ can still be chosen as an objective function in this case.

Proposition 2.2 If $\mathcal{C}_{0}$ is a non-empty class of transient states and $\mathcal{C}_{1}$ is an irreducible class of ergodic states induced by the matrix $P$, then

$$
\operatorname{Tr}[Z] \geq\left|\mathcal{C}_{0}\right|+\frac{\left|\mathcal{C}_{1}\right|-1}{2}>\frac{n-1}{2}
$$

Proof. For $i \in \mathcal{C}_{0}$, we have $\pi_{i}=0$ and thus it follows from the definition of $Z$ (see (4)) that $Z_{i i} \geq 1$. Further, the transition probabilities restricted to $\mathcal{C}_{1}$ constitute a transition matrix of a Markov chain on $\mathcal{C}_{1}$. Note that the latter Markov chain is ergodic. Moreover, according to the definition, the deviation matrix $Z^{(1)}$ of this smaller Markov chain is the same as the corresponding block of the matrix $Z$ of the original chain. Thus, it follows from Proposition 2.1 that

$$
\sum_{i \in \mathcal{C}_{1}} Z_{i i}=\operatorname{Tr}\left[Z^{(1)}\right] \geq \frac{\left|\mathcal{C}_{1}\right|-1}{2},
$$

and the equality is achieved on a Hamiltonian cycle in the sub-graph whose vertices are from $\mathcal{C}_{1}$. Putting everything together, we get

$$
\operatorname{Tr}[Z]=\sum_{i \in \mathcal{C}_{0}} Z_{i i}+\sum_{i \in \mathcal{C}_{1}} Z_{i i} \geq\left|\mathcal{C}_{0}\right|+\frac{\left|\mathcal{C}_{1}\right|-1}{2}
$$


where the last expression is larger than $(n-1) / 2$ simply because $n=\left|\mathcal{C}_{0}\right|+\left|\mathcal{C}_{1}\right|$.

It follows from the above proposition that the chains with one ergodic and one transient class of states can not be candidates for minimizing $\operatorname{Tr}[Z]$. Now, consider a multi-chain with several ergodic classes. Without loss of generality, we unite all transient states in a single transient class. Recall that $G=Z+\Pi$ is the fundamental matrix defined in (1). The following theorem is a generalization of Theorem 4.2 from Hunter and our Proposition 2.1 to the class of all stochastic matrices.

THEOREM 2.1 On a set of all stochastic matrices, the objective function $\operatorname{Tr}[G]$ achieves its minimal value $(n+1) / 2$ at matrix $P$ if and only if $P$ induces a Hamiltonian cycle.

Proof. The argument goes as in the proof of Proposition 2.2. Assume that $P$ induces a Markov chain with $M$ ergodic classes of communicating states $\mathcal{C}_{1}, \ldots, \mathcal{C}_{M}$. For $m=1, \ldots, M$, the transition probabilities restricted to $\mathcal{C}_{m}$ constitute a transition matrix of a Markov chain on $\mathcal{C}_{m}$. The latter smaller Markov chain is ergodic, and its deviation matrix $Z^{(m)}$ is the same as the corresponding block of the matrix $Z$ of the original chain. Moreover, the corresponding block $\Pi^{(m)}$ of $\Pi$ is an ergodic projection of the Markov chain on $\mathcal{C}_{m}$. Thus, it follows from (7) that

$$
\operatorname{Tr}\left[Z^{(m)}+\Pi^{(m)}\right]=\sum_{i \in \mathcal{C}_{m}}\left(Z_{i i}+\Pi_{i i}^{(m)}\right)=\frac{1}{2} \sum_{i \in \mathcal{C}_{m}}\left(c_{i}^{2}+\Pi_{i i}^{(m)}\right)=\frac{1}{2} \sum_{i \in \mathcal{C}_{m}} c_{i}^{2}+\frac{1}{2}, \quad m=1, \ldots, M .
$$

Now, summing over all ergodic classes and transient states and using Proposition 2.2 we obtain

$$
\begin{aligned}
\operatorname{Tr}[G] & =\operatorname{Tr}[Z+\Pi]=\sum_{i \in \mathcal{C}_{0}} Z_{i i}+\sum_{m=1}^{M} \sum_{i \in \mathcal{C}_{m}}\left[Z_{i i}+\Pi_{i i}^{(m)}\right] \\
& =\sum_{i \in \mathcal{C}_{0}} Z_{i i}+\frac{1}{2} \sum_{i=1}^{n} c_{i}^{2}+\frac{1}{2} M \geq\left|\mathcal{C}_{0}\right|+\frac{n}{2}+\frac{M}{2} \geq \frac{n+1}{2} .
\end{aligned}
$$

We see that if all coefficients of variation equal 1 then $\operatorname{Tr}[G]$ is minimized if there is only one ergodic class and no transient state. Then we are back to the irreducible case, and according to Proposition 2.1, the minimal value

$$
(n-1) / 2+\sum_{i=1}^{n} \Pi_{i i}=(n-1) / 2+\sum_{i=1}^{n} \pi_{i}=(n+1) / 2
$$

is achieved iff $P$ induces a Hamiltonian cycle.

From the above theorem we conclude that actually the trace of a fundamental matrix $G$ rather than the trace of $Z$ is the proper objective function. Simply, for irreducible Markov chains, these two objective functions are equivalent.

3. Trace versus diagonal element. In the previous works Borkar et al. [7, 8], Ejov et al. [12], the Hamiltonian cycle problem was formulated as an optimization problem, where the objective function was the first diagonal element of a fundamental matrix, $G_{11}$, rather than the trace $\operatorname{Tr}[G]$. The next proposition shows that these two criteria are equivalent on the class $\mathcal{D S}$ of doubly stochastic Markov chains. We remind that for any doubly stochastic Markov chain, the invariant distribution is uniform and thus there can not be any transient state.

Proposition 3.1 On a set of doubly stochastic matrices, minimizing $G_{i i}$ for some given $i \in \mathcal{S}$ is equivalent to minimizing $\operatorname{Tr}[G]$.

ProOf. Since all states are recurrent, any state $i=1, \ldots, n$ belongs to one of the disjoint ergodic classes $\mathcal{C}_{1}, \ldots, \mathcal{C}_{M}$, each of which can be seen as a separate doubly stochastic Markov chain. Then assuming $i \in \mathcal{C}_{1}$, from (7) we get

$$
G_{i i}=Z_{i i}+\Pi_{i i}=\frac{1}{2}\left(c_{i}^{2}+\frac{1}{\left|\mathcal{C}_{1}\right|}\right)
$$

which is clearly minimized on a Hamiltonian cycle with $c_{i}^{2}=1$ and $1 /\left|\mathcal{C}_{1}\right|=1 / n$. Now, let $P$ be doubly stochastic and irreducible so that $\pi_{i}=1 / n$, and assume that $P$ minimizes $c_{i}^{2}$, that is, $c_{i}^{2}=1$. Then, with 
probability $1, \tau_{i}$ equals its expectation $n$. Since $\tau_{i}$ is deterministic, each state has to be visited exactly once on the way from $i$ back to $i$. Moreover, the order of visiting the states must be fixed, otherwise, there will be a possibility to shorten or extend the cycle. Thus, we conclude that the condition $c_{i}^{2}=1$ in a doubly stochastic chain ensures the Hamiltonian cycle and, consequently, $c_{j}^{2}=1$ for all $j \in \mathcal{S}$. Hence, according to Proposition 2.1, $\operatorname{Tr}[G]=\operatorname{Tr}[Z]+1$ is also minimized on this matrix.

Now, consider an arbitrary irreducible stochastic matrix $P$. Since $c_{1}^{2} \geq 1$, it follows that $G_{11}=$ $Z_{11}+\pi_{1}=(1 / 2)\left(c_{1}^{2}+\pi_{1}\right)>1 / 2+1 /(2 n)$ if $\pi_{1}>1 / n$. Assume now that $\pi_{1}<1 / n$. Then the average cycle length between two successive visits to state 1 is greater than $n$, and thus the cycle typically has loops implying that the cycle length is rather variable. The question is whether the gain in $\pi_{1}$ can compensate for the increased coefficient of variation when minimizing $G_{11}$. We partially resolve this problem by considering a particular class of Markov chains that was described in Hunter [19] as 'Cycle drift'.

The Cycle drift is defined as follows. At each state $i=1, \ldots, n$, with probability $p_{i}$, the process follows a cyclic path $1 \rightarrow 2 \rightarrow \cdots \rightarrow n \rightarrow 1$. With complementary probability, $1-p_{i}$, the process makes a self-loop and stays in state $i$. A Hamiltonian cycle is a special case of a Cycle drift where a probability of a self-loop is zero in each state. The following proposition holds.

Proposition 3.2 On a class of Markov chains inducing a Cycle drift, the minimum of $G_{11}$ is equal to $1 / 2+1 /(2 n)$ and is achieved on a Hamiltonian cycle.

Proof. Assume that a Cycle drift has a stationary distribution $\pi$ with $\pi_{1} \leq 1 / n$. Define a renewal cycle as a time between two subsequent transitions from state $n$ to state 1 . Let $C$ be the average length of the renewal cycle. Since the length of stay in each state is geometric, we have

$$
C=\sum_{i=1}^{n} 1 / p_{i} \geq n .
$$

Observe that the beginning of each renewal cycle constitutes a regenerative epoch of our Markov process, and hence the Renewal Theory implies that

$$
\pi_{i}=\frac{\mathbb{E}[\text { time in state } i \text { on a cycle }]}{\mathbb{E}[\text { average cycle length }]}=\frac{1 / p_{i}}{C} \geq \frac{1}{C}, \quad i=1, \ldots, n .
$$

Now we use the formula $Z_{11}=\pi_{1} \mathbb{E}_{\pi}\left[T_{1}\right]$ to compute $Z_{11}$. For $\mathbb{E}_{\pi}\left[T_{1}\right]$, we obtain the following:

$$
\begin{aligned}
\mathbb{E}_{\pi}\left[T_{1}\right] & =0 \cdot \pi_{1}+\sum_{i=2}^{n} \pi_{i} \sum_{j=i}^{n} 1 / p_{j}=\frac{1}{C} \sum_{i=2}^{n}\left(1 / p_{i}\right) \sum_{j=i}^{n} 1 / p_{j} \\
& =\frac{1}{2 C}\left(\sum_{i=2}^{n} 1 / p_{i}\right)^{2}+\frac{1}{2 C} \sum_{i=2}^{n}\left(1 / p_{i}\right)^{2}=\frac{1}{2 C}\left(C-1 / p_{1}\right)^{2}+\frac{1}{2 C} \sum_{i=2}^{n}\left(1 / p_{i}\right)^{2} .
\end{aligned}
$$

According to the Jensen's inequality, the second term in the last expression is minimized when $1 / p_{i}=$ constant for $i=2, \ldots, n$. Then we have

$$
1 / p_{i}=\left(C-1 / p_{1}\right) /(n-1),
$$

and (9) reduces to

$$
\mathbb{E}_{\pi}\left[T_{1}\right]=\frac{1}{2 C}\left(C-1 / p_{1}\right)^{2}+\frac{1}{2 C} \frac{\left(C-1 / p_{1}\right)^{2}}{n-1}=\frac{C}{2}\left(1-\pi_{1}\right)^{2} \frac{n}{n-1} .
$$

Now we have to find out which value of $C \geq n$ and which $\pi_{1} \in[1 / C, 1 / n]$ minimize the function

$$
Z_{11}+\pi_{1}=\pi_{1} \mathbb{E}_{\pi}\left[T_{1}\right]+\pi_{1}=\frac{C}{2}\left(1-\pi_{1}\right)^{2} \pi_{1} \frac{n}{n-1}+\pi_{1} .
$$

For $n \geq 3$, it is easy to check that the expression on the right-hand side is increasing in $\pi_{1}$ for $\pi_{1} \leq 1 / n$ and thus, for minimizing $Z_{11}+\pi_{1}$ with given $C$, we have to choose the smallest value $\pi_{1}=1 / C$. Substituting this in (10), we get

$$
Z_{11}+\pi_{1}=\frac{1}{2}\left(1-\frac{1}{C}\right)^{2} \frac{n}{n-1}+\frac{1}{C} .
$$


The expression on the right-hand side is minimized at $C=n$. For $n=2$, the right-hand side of (10) achieves its minimal value when $\pi_{1}=1 / 2$ and $C=2$. Thus, the $Z_{11}+\pi_{1}$ is minimized when $p_{i}=1$, $i=1, \ldots, n$, which corresponds to the Hamiltonian cycle.

Hoping that Proposition 3.2 can be generalized to the class of all stochastic transition matrices, we conclude this section with the following conjecture.

Conjecture 1 Minimizing $\operatorname{Tr}[G]$ is equivalent to minimizing $G_{11}$. In both cases, the minimum is achieved at a Hamiltonian matrix.

4. Benefits of perturbations. In Section 2 we claimed that the Hamiltonian cycle problem can be resolved by minimizing $\operatorname{Tr}[G]=\operatorname{Tr}[Z+\Pi]$ on a class of all stochastic matrices corresponding to a given graph. This formulation however is not very practical for several reasons. First, the objective function is difficult to compute. There is no guarantee that series in (4) converge fast enough. The alternative way of determining $G$ by using the matrix inverse (1) might be computationally demanding for large graphs, and it requires the knowledge of $\Pi$. Second, a deviation matrix turns out to be very sensitive to small changes in the transition matrix $P$. Such sensitivity finds its explanation in the theory of singularly perturbed Markov chains. A perturbation of a Markov chain is singular if it alters the ergodic structure of the chain, for instance, if a reducible chain becomes irreducible, or some transient states become recurrent, or the number of ergodic classes changes. Assume for example that the transition matrix $P$ has $M$ ergodic classes. Then by adding small but positive transition probabilities between two states in different classes, we change the number of classes which leads to drastic changes in the deviation matrix (see Avrachenkov and Lasserre [4]). In a special case of symmetric linear perturbation, we will observe this phenomenon in Proposition 4.1 below. For more details on singularly perturbed Markov chains we refer to Avrachenkov and Lasserre [4] and other works by Avrachenkov and co-authors.

Given the sensitivity of the deviation matrix to singular perturbations, it would be convenient to preserve the ergodic structure of the chain while changing the transition probabilities. Ideally, we would like to keep the transition matrix irreducible but imposing irreducibility on $P$ is inconvenient as discussed in Section 2. A powerful way to guarantee irreducibility is to introduce a symmetric linear perturbation as in Ejov et al. [12]. Let $J$ be the matrix consisting only of units. Then for any $\varepsilon \in(0,1)$ we define

$$
P(\varepsilon)=[1-\varepsilon] P+\varepsilon \frac{1}{n} J .
$$

The transition matrix $P(\varepsilon)$ induces a Markov chain that at each step follows $P$ with probability $(1-\varepsilon)$ and picks the next state uniformly at random with probability $\varepsilon$. The symmetric perturbation $\varepsilon \frac{1}{n} J$ ensures irreducibility and aperiodicity of $P(\varepsilon)$. This implies that the corresponding deviation matrix $Z(\varepsilon)$ is much less sensitive to changes in $P$ than the original deviation matrix $Z$. We note that since $P(\varepsilon)$ is irreducible, we have

$$
\operatorname{Tr}[G(\varepsilon)]=\operatorname{Tr}[Z(\varepsilon)+\Pi(\varepsilon)]=\operatorname{Tr}[Z(\varepsilon)]+1,
$$

where $G(\varepsilon)$ is the fundamental matrix of $P(\varepsilon)$. In what follows we will operate with $Z(\varepsilon)$ rather than $G(\varepsilon)$ because $\operatorname{Tr}[Z(\varepsilon)]$ has a natural probabilistic interpretation via the Random Target Lemma. The results obtained for $\operatorname{Tr}[Z(\varepsilon)]$ apply to $\operatorname{Tr}[G(\varepsilon)]$ straightforwardly via (12).

Observe that if $P$ is reducible then the symmetric linear perturbation is singular. Thus, the results from perturbation analysis suggest that for any $i \in \mathcal{S}$, the quantity $Z_{i i}(\varepsilon)$ goes to $+\infty$ as $\varepsilon$ approaches 0 . Therefore, when $\varepsilon$ is small enough, all reducible chains will have a higher value of $\operatorname{Tr}[Z(\varepsilon)]$ than the perturbed Hamiltonian path. Hence, a small perturbation helps to filter out reducible matrices when minimizing $\operatorname{Tr}[Z(\varepsilon)]$. This argument is formalized in the next proposition.

Proposition 4.1 (i) Assume that $P$ is reducible. Then $\operatorname{Tr}[Z(\varepsilon)] \geq 1 /(n \varepsilon)$; (ii) If $P$ induces a Hamiltonian cycle then for all $\varepsilon<1 / n$,

$$
\operatorname{Tr}[Z(\varepsilon)] \leq \frac{n-1}{2}+\frac{(n-1)^{2}}{2} \varepsilon
$$

Proof. To prove (i), note that a reducible matrix $P$ induces at least one class $\mathcal{C}(\varepsilon)$ (transient or ergodic) such that in the perturbed chain, $\sum_{j \in \mathcal{C}(\varepsilon)} \pi_{j}(\varepsilon) \geq 1 / n$. Now, choose some state $i \notin \mathcal{C}(\varepsilon)$ as an 
initial state. In order to reach $\mathcal{C}(\varepsilon)$ starting from $i$, at least one $\varepsilon$-transition has to be made, so the mean hitting time is not smaller than $1 / \varepsilon$. Hence, according to the Random Target Lemma,

$$
\operatorname{Tr}[Z(\varepsilon)]=\sum_{j} \pi_{j}(\varepsilon) \mathbb{E}_{i} T_{j}(\varepsilon) \geq \sum_{j \in \mathcal{C}(\varepsilon)} \pi_{j}(\varepsilon) \mathbb{E}_{i} T_{j}(\varepsilon) \geq \pi_{\mathcal{C}(\varepsilon)}(\varepsilon)(1 / \varepsilon) \geq 1 /(n \varepsilon) .
$$

For (ii), consider the perturbation of the matrix inducing a Hamiltonian cycle. Applying the Random Target Lemma and taking into account that $\pi_{j}(\varepsilon)=1 / n$ because the perturbed matrix is doubly stochastic, we get

$$
\operatorname{Tr}[Z(\varepsilon)]=\sum_{j} \pi_{j}(\varepsilon) \mathbb{E}_{1} T_{j}(\varepsilon)=\frac{1}{n} \sum_{j} \mathbb{E}_{1} T_{j}(\varepsilon) .
$$

Given that no $\varepsilon$-transition occurs on the way from 1 to $j$, the last expression equals $(n-1) / 2$. The chance of at least one $\varepsilon$-transition on the way from 1 to $j$ is at most $\left(1-(1-\varepsilon)^{n-1}\right)$. Further, after an $\varepsilon$-transition, the state $j$ has to be reached starting from some new initial distribution $\mu$. Using again the Random Target Lemma, we obtain

$$
\begin{aligned}
\operatorname{Tr}[Z(\varepsilon)] & \leq \frac{n-1}{2}+\left(1-\left((1-\varepsilon)^{n-1}\right) \sum_{j} \pi_{j}(\varepsilon) \mathbb{E}_{\mu} T_{j}(\varepsilon)\right. \\
& =\frac{n-1}{2}+\left(1-\left((1-\varepsilon)^{n-1}\right) \operatorname{Tr}[Z(\varepsilon)] .\right.
\end{aligned}
$$

It follows that

$$
\begin{aligned}
\operatorname{Tr}[Z(\varepsilon)] \leq \frac{n-1}{2}(1-\varepsilon)^{-(n-1)} & =\frac{n-1}{2}\left[1+(n-1) \varepsilon+O\left(\varepsilon^{2}\right)\right] \\
& \leq \frac{n-1}{2}+\frac{(n-1)^{2}}{2} \varepsilon, \quad \text { if } \quad \varepsilon<1 / n
\end{aligned}
$$

Proposition 4.1 implies that if $\varepsilon<1 / n^{2}$ then for any multi-chain matrix $P$, we have $\operatorname{Tr}[Z(\varepsilon)] \geq n$ whereas for a Hamiltonian matrix, $\operatorname{Tr}[Z(\varepsilon)]$ is not greater than $n / 2$. Thus, if a Hamiltonian cycle exists then for $\varepsilon<1 / n^{2}$, a multi-chain matrix can not be a candidate for minimizing $\operatorname{Tr}[Z(\varepsilon)]$. The disadvantage of this approach however is that a very small perturbation does not resolve the above mentioned issues on computation and robustness of the objective function. In Section 5 we will show that on the class of doubly stochastic matrices, any $\varepsilon \in(0,1)$ can be used for solving the Hamiltonian cycle problem.

The matrices with symmetric linear perturbation have recently received a massive attention in Computer Science literature, mainly because this sort of matrices is used in the Google PageRank algorithm that determines popularity of Web pages. The PageRank is defined as a stationary distribution of a Markov chain on a set of Web pages. This Markov chain serves as an elementary model of a surfing process. At each step, with probability $(1-\varepsilon)$, a surfer follows a randomly chosen out-going hyperlink of a current page, and with probability $\varepsilon$, the surfer is bored and picks a new page on the Web at random. Clearly, a jump to a random page with probability $\varepsilon$ is exactly the symmetric linear perturbation of a random walk on the Web graph, and the PageRank vector is the stationary vector of $P(\varepsilon)$. Google originally chose $\varepsilon=0.15$. After introducing of PageRank by Brin and Page [9], a lot of work has been done on analyzing the formula and on determining the exact meaning of the parameter $\varepsilon$. We refer to Langville and Meyer [20] for an excellent survey of a PageRank research. A number of explicit results are available on sensitivity of PageRank to small changes in the Web (e.g. Avrachenkov and Litvak [3], Bianchini et al. [6]), on the influence of the 'damping factor' $\varepsilon$, and on the relation between the PageRank vector and the Web structure. However, a lion share of papers is devoted to enhancing the PageRank computation. A comprehensive overview on this topic can be found in Berkhin [5], Langville and Meyer [20].

It was shown in Langville and Meyer [20] and other PageRank literature that the powers $P^{t}(\varepsilon)$ converge to $\Pi(\varepsilon)$ at least as fast as $(1-\varepsilon)^{t}$ goes to zero as $t \rightarrow \infty$. More specifically, it was proved in Haveliwala and Kamvar [18] that the absolute value of the second eigenvalue of $P(\varepsilon)$ is at most $(1-\varepsilon)$. Below we prove a stronger result for a doubly stochastic matrix $P$ by stating the following simple linear algebraic fact.

Lemma 4.1 If $1, \lambda_{i}, i=1, \ldots, n-1$ are the eigenvalues (repeated with their multiplicities) of the doubly stochastic Markov chain $P$ then the eigenvalues of $P(\varepsilon)$ in $(11)$ are $1,(1-\varepsilon) \lambda_{i}, i=1, \ldots, n-1$. 
Proof. Since $P$ is doubly stochastic, then $J P=J$ and, hence, the $(n-1)$-dimensional null-space ker $J$ is also $P$-invariant. The complimentary (to ker $J$ ) vector $\mathbf{1}=[1, \ldots, 1]$ remains, obviously, the eigenvector of $P(\varepsilon)$ of the eigenvalue 1 . The remaining eigenvectors of $P(\varepsilon)$ can be chosen from the ker $J$ and, therefore, they have to be the eigenvectors of the matrix $(1-\varepsilon) P$ which has eigenvalues $(1-\varepsilon) \lambda_{i}$, $i=1, \ldots, n-1$, accordingly.

The matrix $P(\varepsilon)$ is aperiodic, hence, the corresponding deviation matrix $Z(\varepsilon)$ is defined by (3). Moreover, the results on the eigenvalues imply that the $t$-th term of the series in (3) is of the order at most $(1-\varepsilon)^{t}$, and thus the convergence improves significantly if $\varepsilon$ is not very small. Clearly, numerical methods developed for computing PageRank can be applied for computing the deviation matrix $Z(\varepsilon)$. Further discussion of computational issues is however beyond the scope of this paper.

5. Doubly stochastic matrices with symmetric linear perturbation. Assume that $P$ is doubly stochastic. Then $P(\varepsilon)$ in (11) is also doubly stochastic, and we get an elementary relation

$$
G_{11}(\varepsilon)=Z_{11}(\varepsilon)+\pi_{1}(\varepsilon)=Z_{11}(\varepsilon)+1 / n .
$$

It was shown in Borkar et al. [8] that on the class of doubly stochastic matrices, $G_{11}(\varepsilon)$ is minimized at the matrix inducing a Hamiltonian cycle for all $\varepsilon$ in a small neighborhood of zero. The proof in Borkar et al. [8] heavily relies on linear algebraic methods. In this section, we use a probabilistic approach to show that a Hamiltonian cycle ensures a minimal value of $G_{11}(\varepsilon)$ for any $\varepsilon \in(0,1)$. This is a considerable step towards applicability of the results in this line of research, because, as we discussed in the previous section, a reasonably large value of $\varepsilon$ helps to resolve some serious computational issues. Besides, this surprising generalization is interesting from pure mathematical point of view.

From now on, we will use an upper index $(H)$ for characteristics related to the Hamiltonian matrix, and upper index $(P)$ for respective characteristics related to an arbitrary matrix $P$. The additional argument $\varepsilon$ as in $P(\varepsilon)$ indicates that the mentioned quantity corresponds to the matrix with symmetric linear perturbation.

THEOREM 5.1 For any admissible $\varepsilon \in(0,1)$ and any doubly stochastic matrix $P$ holds

$$
Z_{i i}^{(H)}(\varepsilon) \leq Z_{i i}^{(P)}(\varepsilon), \quad i=1, \ldots, n,
$$

with equality if and only if $P$ induces a Hamiltonian cycle.

Proof. Fix an arbitrary state, say, state 1 . We first consider matrices $H$ and $P$ without perturbation and prove that if the process starts from the uniform distribution $\pi$ then $T_{1}^{(H)}$ is stochastically smaller than $T_{1}^{(P)}$. Formally, we want to show that

$$
\mathbb{P}_{\pi}\left[T_{1}^{(H)} \geq k\right] \leq \mathbb{P}_{\pi}\left[T_{1}^{(P)} \geq k\right], \quad k=1,2, \ldots
$$

Since for the Hamiltonian cycle we have $\mathbb{P}_{\pi}\left[T_{1}^{(H)}=k\right]=1 / n$ for $k=0, \ldots, n-1$, it is sufficient to prove that $\mathbb{P}_{\pi}\left[T_{1}^{(P)}=k\right] \leq 1 / n, k=0, \ldots, n-1$. For $k=0$, we obviously have $\mathbb{P}_{\pi}\left[T_{1}^{(P)}=0\right]=\pi_{1}=1 / n$. Next, denoting by $\left(X_{t}^{(P)}\right)$ the Markov chain induced by $P$, for $k=1, \ldots, n-1$, we get

$$
\begin{aligned}
\mathbb{P}_{\pi}\left[T_{1}^{(P)}=k\right] & =\mathbb{P}_{\pi}\left[X_{k}^{(P)}=1 ; X_{t}^{(P)} \neq 1, t=0, \ldots, k-1\right] \\
& \leq \mathbb{P}_{\pi}\left[X_{k}^{(P)}=1\right]=\pi_{1}=1 / n, \quad k=0, \ldots, n-1 .
\end{aligned}
$$

Let us now show that

$$
\mathbb{E}_{\pi}\left(T_{1}^{(H)}(\varepsilon)\right) \leq \mathbb{E}_{\pi}\left(T_{1}^{(P)}(\varepsilon)\right)
$$

Since the stationary distribution is uniform for both $H(\varepsilon)$ and $P(\varepsilon)$, (14) will follow from (17) and (5).

In order to prove (17), consider a random walk induced by the perturbed matrix $P(\varepsilon)$. Assume that the initial distribution of such random walk is $\pi$, i.e. uniform. At each step, with probability $(1-\varepsilon)$, the random walk makes a step according to the matrix $P$, and with probability $\varepsilon$, it makes a jump to a random state. After such a jump, the random walk probabilistically restarts itself, so we have a renewal process where random jumps constitute the renewal events.

Let $N$ be the number of renewals (including the ' 0 -th'renewal at time 0 ) before the process reaches state 1 . Clearly, $N$ is a stopping time, where the process stops once state 1 is reached. Since the random 
jump can occur at every step with equal probability, we can formally write it as follows. Denote by $Y$ a geometric random variable with parameter $\varepsilon$. Let $Y_{0}, Y_{1}, \ldots$ be a sequence of independent random variables distributed as $Y$. Further, for $m \geq 0$, let $T_{1, m}$ denote the time needed to reach state 1 after the $m$-th renewal. Due to the strong Markov property, all $T_{1, m}$ 's are identically distributed, and, provided that the initial distribution is uniform, $T_{1,0}$ is equivalent to $T_{1}$. In this setup, we write

$$
N=1+\min \left\{m \geq 0: T_{1, m}<Y_{m}\right\} .
$$

Then, according to the Wald's equation, we have

$$
\mathbb{E}_{\pi}\left[T_{1}(\varepsilon)\right]=\mathbb{E}_{\pi}[N] \mathbb{E}_{\pi}\left[\min \left\{T_{1}, Y\right\}\right]
$$

Clearly, $N$ is a geometric random variable, and

$$
\mathbb{E}_{\pi}[N]=1 / \mathbb{P}_{\pi}\left[T_{1}<Y\right]
$$

Moreover, since $\mathbb{P}_{\pi}\left[T_{1}^{(H)}<k\right] \geq \mathbb{P}_{\pi}\left[T_{1}^{(P)}<k\right]$ for $k=1,2, \ldots$, we have

$$
\mathbb{P}_{\pi}\left[T_{1}^{(H)}<Y\right]=\sum_{k=1}^{\infty} \mathbb{P}[Y=k] \mathbb{P}_{\pi}\left[T_{1}^{(H)}<k\right] \geq \sum_{k=1}^{\infty} \mathbb{P}[Y=k] \mathbb{P}_{\pi}\left[T_{1}^{(P)}<k\right]=\mathbb{P}_{\pi}\left[T_{1}^{(P)}<Y\right]
$$

It follows that

$$
\mathbb{E}_{\pi}\left[N^{(H)}\right] \leq \mathbb{E}_{\pi}\left[N^{(P)}\right]
$$

for any doubly stochastic matrix $P$. Furthermore,

$$
\mathbb{E}_{\pi}\left[\min \left\{T_{1}^{(H)}, Y\right\}\right]=\mathbb{E}_{\pi}\left[\sum_{k=1}^{Y} \mathbb{P}_{\pi}\left[T_{1}^{(H)} \geq k\right]\right] \leq \mathbb{E}_{\pi}\left[\sum_{k=1}^{Y} \mathbb{P}_{\pi}\left[T_{1}^{(P)} \geq k\right]\right]=\mathbb{E}_{\pi}\left[\min \left\{T_{1}^{(P)}, Y\right\}\right] .
$$

Equation (17) now follows from (18), (20) and (21). Combining (17) and (5) we obtain (14).

It remains to show that the equality in (14) is possible iff $P$ induces a Hamiltonian cycle. For that, we only need to prove that for any doubly stochastic non-Hamiltonian $P$, (15) becomes a strict inequality at least for some $k>0$. If $P$ is reducible, then this is obvious because in this case $\mathbb{P}_{\pi}\left[T_{i}=+\infty\right]>0$ for all $i=1, \ldots, n$. For irreducible $P$, consider an arbitrary state, say state 1 . If the cycle length $\tau_{1}^{(P)}$ is random then $\mathbb{P}\left[\tau_{1}^{(P)}>n\right]>0$. Thus, there exists a state $j$ such that $\mathbb{P}_{j}\left[T_{1}^{(P)}>n-1\right]>0$, which implies $\mathbb{P}_{\pi}\left[T_{1}^{(P)}>n-1\right]>0$, and the desired strict inequality for some $k=1, \ldots, n-1$ follows from (16). Furthermore, if $\tau_{1}^{(P)}$ is a constant and $P$ is doubly stochastic, then it follows from the proof of Proposition 3.1 that $P$ induces a Hamiltonian cycle. This completes the proof of the theorem.

The following corollary is an immediate consequence of Theorem 5.1 combined with, respectively, (13), the definition of the trace, and (12).

Corollary 5.1 For any admissible $\varepsilon \in(0,1)$ and any doubly stochastic matrix $P$ holds

$$
\begin{gathered}
G_{i i}^{(H)}(\varepsilon) \leq G_{i i}^{(P)}(\varepsilon), \quad i=1, \ldots, n, \\
\operatorname{Tr}\left[Z^{(H)}(\varepsilon)\right] \leq \operatorname{Tr}\left[Z^{(P)}(\varepsilon)\right],
\end{gathered}
$$

and

$$
\operatorname{Tr}\left[G^{(H)}(\varepsilon)\right] \leq \operatorname{Tr}\left[G^{(P)}(\varepsilon)\right] .
$$

The equality in (22) - (24) holds if and only if $P$ induces a Hamiltonian cycle.

In general case $P \in \mathcal{F}$, the argument does not work, and actually the statement of Theorem 5.1 does not hold for all $\varepsilon \in(0,1)$ as the following example illustrates. Let $\mathcal{G}$ be a Hamiltonian graph on 4 nodes with the adjacency matrix that we also denote by $\mathcal{G}$ be

$$
\mathcal{G}=\left[\begin{array}{llll}
0 & 1 & 1 & 1 \\
1 & 0 & 1 & 0 \\
1 & 0 & 0 & 1 \\
1 & 0 & 0 & 0
\end{array}\right]
$$


Consider the transition matrix

$$
P=\left[\begin{array}{cccc}
0 & \frac{1}{3} & \frac{1}{3} & \frac{1}{3} \\
1 & 0 & 0 & 0 \\
1 & 0 & 0 & 0 \\
1 & 0 & 0 & 0
\end{array}\right]
$$

and its perturbation $P(\varepsilon)$ of the form (11). Here the value $Z_{11}(\varepsilon)$ is minimized on a Hamiltonian matrix for small values of $\varepsilon$. For example, for $\varepsilon=0.24$ we have $Z_{11}^{(P)}(\varepsilon) \approx 0.784$ and $Z_{11}^{(H)}(\varepsilon) \approx 0.708$. For large $\varepsilon$, the situation is different: choosing $\varepsilon=0.76$ we get $Z_{11}^{(P)}(\varepsilon) \approx 0.903$, which is smaller than $Z_{11}^{(H)}(\varepsilon) \approx 0.924$.

We admit however that we could not construct an example of a stochastic matrix $P$ such that $\operatorname{Tr}\left[Z^{(P)}(\varepsilon)\right]$ is smaller than $\operatorname{Tr}\left[Z^{(H)}(\varepsilon)\right]$ for at least some $\varepsilon \in(0,1)$. For instance, in the example above, we get $\operatorname{Tr}\left[Z^{(P)}(\varepsilon)\right] \approx 3.568, \operatorname{Tr}\left[Z^{(H)}(\varepsilon)\right] \approx 2.836$ for $\varepsilon=0.24$, and $\operatorname{Tr}\left[Z^{(P)}(\varepsilon)\right] \approx 3.806, \operatorname{Tr}\left[Z^{(H)}(\varepsilon)\right] \approx 3.698$ for $\varepsilon=0.76$. Moreover, in this example, $G_{11}^{(P)}(\varepsilon)$ also happens to be larger than $G_{11}^{(H)}(\varepsilon)$ for all admissible $\varepsilon$. It is tempting to hypothesize that (23), (24), and maybe even (22) might hold on the set $\mathcal{F}$ of stochastic matrices for any $\varepsilon \in(0,1)$, even though (14) fails for some of the states when $\varepsilon$ is large enough. Proving or disproving this conjecture remains an intriguing open problem.

6. Conclusions and further research. A number of functionals stemming from connections with Markov chains can be used in optimization problems that identify Hamiltonian cycles. We refer to Filar [14] for a comprehensive overview. In the present paper we studied in detail the functional $\operatorname{Tr}[G]$. Compared to the functional $G_{11}$ addressed in the previous work, $\operatorname{Tr}[G]$ simplifies the analysis and allows for various probabilistic interpretations. In particular, we have shown that Hamiltonian cycles minimize $\operatorname{Tr}[G]$ over a set of all stochastic matrices while a similar result for $G_{11}$ could not yet be proved.

Nevertheless, the functionals $\operatorname{Tr}[G]$ and $G_{11}$ are similar not only from algebraic point of view but also probabilistically, because they both reflect the variances of return times in a corresponding Markov chain. Thus, the Hamiltonian matrix is optimal in a sense that it minimizes variability. Moreover, we conjecture that minimizing $\operatorname{Tr}[G]$ is in fact equivalent to minimizing $G_{11}$, despite of the possibility to choose state 1 so that $G_{11}$ is minimized at the expense of other states.

Computationally, $\operatorname{Tr}[G(\varepsilon)]$ looks like a suitable objective function as it allows all admissible values for the perturbation parameter over a set $\mathcal{D S}$ of doubly stochastic matrices. More research is needed to find out whether this will lead to efficient methods for solving HCP, at least for some classes of graphs. The novelty of our approach in analyzing $\operatorname{Tr}[G(\varepsilon)]$ and $G_{11}(\varepsilon)$ is in the decomposition of the perturbed Markov chain into an original chain and random jumps that constitute renewal instants. A new challenge is to extend this line of argument to other functionals and beyond the $\mathcal{D S}$ set.

In future, it will be also interesting to look for new, completely different functionals that do not entirely rely on minimizing variability. Another direction is to find a subclass of matrices where minimizing $G_{11}$ or $\operatorname{Tr}[G]$ is easy. For instance, an appealing special case is the set $\mathcal{D} S S$ of symmetric doubly stochastic matrices. It was shown in Borkar et al. [8] that $G_{11}$ is strictly convex over $\mathcal{D} S S$. However, it is not clear whether a convex program for $G_{11}$ over $\mathcal{D} S S$ can be related to the Hamiltonian cycle problem.

Acknowledgment. Nelly Litvak gratefully acknowledges the financial support of the Netherlands Organization for Scientific Research (NWO) under the Meervoud grant 632.002.401. Vladimir Ejov acknowledges the support of the Australian Research Council grants DP0666632 and LX0560049. We are indebted to J. Filar for the initial inspiration and many useful discussions during the conduct of the work.

\section{References}

[1] D. Aldous and J. Fill. Reversible Markov chains and random walks on graphs. Available at http://www.stat.berkeley.edu/users/aldous/RWG/book.html, 2002.

[2] M. Andramonov, J. Filar, P. Pardalos, and A. Rubinov. Hamiltonian cycle problem via Markov chains and min-type approaches. In Approximation and complexity in numerical optimization (Gainesville, FL, 1999), volume 42 of Nonconvex Optim. Appl., pages 31-47. Kluwer Acad. Publ., Dordrecht, 2000. 
[3] K. Avrachenkov and N. Litvak. The effect of new links on Google PageRank. Stoch. Models, 22(2): 319-331, 2006. ISSN 1532-6349.

[4] K. E. Avrachenkov and J. B. Lasserre. The fundamental matrix of singularly perturbed Markov chains. Adv. in Appl. Probab., 31(3):679-697, 1999. ISSN 0001-8678.

[5] P. Berkhin. A survey on PageRank computing. Internet Math., 2:73-120, 2005.

[6] M. Bianchini, M. Gori, and F. Scarselli. Inside PageRank. ACM Trans. Inter. Tech., 5(1):92-128, 2005. ISSN 1533-5399.

[7] V. S. Borkar, V. Ejov, and J. A. Filar. Directed graphs, Hamiltonicity and doubly stochastic matrices. Random Structures Algorithms, 25(4):376-395, 2004. ISSN 1042-9832.

[8] V. S. Borkar, V. Ejov, and J. A. Filar. On the Hamiltonicity gap and doubly stochastic matrices. Technical report, University of South Australia, 2006. Available at http://www.unisanet.unisa.edu.au/staff/VladimirEjov/borkar_gap.pdf.

[9] S. Brin and L. Page. The anatomy of a large-scale hypertextual web search engine. Computer Networks and ISDN Systems, 33:107-117, 1998.

[10] M. Chen and J. A. Filar. Hamiltonian cycles, quadratic programming, and ranking of extreme points. In Recent advances in global optimization (Princeton, NJ, 1991), Princeton Ser. Comput. Sci., pages 32-49. Princeton Univ. Press, Princeton, NJ, 1992.

[11] V. Ejov, J. Filar, and J. Gondzio. An interior point heuristic for the Hamiltonian cycle problem via Markov decision processes. J. Global Optim., 29(3):315-334, 2004. ISSN 0925-5001.

[12] V. Ejov, J. A. Filar, and M.-T. Nguyen. Hamiltonian cycles and singularly perturbed Markov chains. Math. Oper. Res., 29(1):114-131, 2004. ISSN 0364-765X.

[13] E. A. Feinberg. Constrained discounted Markov decision processes and Hamiltonian cycles. Math. Oper. Res., 25(1):130-140, 2000. ISSN 0364-765X.

[14] J. A. Filar. Controlled Markov chains, graphs \& Hamiltonicity. Technical report, University of South Australia, 2007. In preparation.

[15] J. A. Filar and D. Krass. Hamiltonian cycles and Markov chains. Math. Oper. Res., 19(1):223-237, 1994. ISSN 0364-765X.

[16] J. A. Filar and J. B. Lasserre. A non-standard branch and bound method for the Hamiltonian cycle problem. In Proceedings of the 1999 International Conference on Computational Techniques and Applications (Canberra), volume 42, pages C586-C607, 2000.

[17] J. A. Filar and K. Liu. Hamiltonian cycle problem and singularly perturbed Markov decision process. In Statistics, probability and game theory, volume 30 of IMS Lecture Notes Monogr. Ser., pages 45-63. Inst. Math. Statist., Hayward, CA, 1996.

[18] T. H. Haveliwala and S. D. Kamvar. The second eigenvalue of the Google matrix. Technical report, Stanford University, 2003.

[19] J. J. Hunter. Mixing times with applications to perturbed Markov chains. Linear Algebra Appl., 417 (1):108-123, 2006. ISSN 0024-3795.

[20] A. N. Langville and C. D. Meyer. Deeper inside PageRank. Internet Math., 1:335-380, 2003.

[21] L. Lovász and P. Winkler. Mixing times. In Microsurveys in discrete probability (Princeton, NJ, 1997), volume 41 of DIMACS Ser. Discrete Math. Theoret. Comput. Sci., pages 85-133. Amer. Math. Soc., Providence, RI, 1998.

[22] S. M. Ross. Stochastic processes. Wiley Series in Probability and Statistics: Probability and Statistics. John Wiley \& Sons Inc., New York, second edition, 1996. ISBN 0-471-12062-6. 